\title{
On the Möbius geometry of Euclidean triangles
}

\author{
Udo Hertrich-Jeromin, Alastair King and Jun O’Hara
}

Udo Hertrich-Jeromin obtained his Doctorate in 1994 and did his Habilitation in 2002, both at the Technical University in Berlin. He is now a professor at the Vienna University of Technology after having worked for nine years at the University of Bath as a lecturer. His main research interests are in smooth and discrete submanifolds in sphere geometries.

Alastair King obtained his PhD from the University of Oxford in 1989 and is now a Professor of Mathematics at the University of Bath. His main research interests are algebraic geometry and representation theory.

Jun O'Hara obtained his PhD from the University of Toyko in 1991. He is now Associate Professor at the Tokyo Metropolitan University. His main mathematical interests are in knot energies and the conformal geometry of curves and surfaces.

\section{Introduction}

We take a Möbius geometric look at the in- and ex-centres of a triangle in the Euclidean plane: at first this appears to be an absurd endeavour as the centres of circles are not Möbius geometrically attached to their circles. But surprisingly, it turns out that there is a symmetric, Möbius geometric construction of the four in- and ex-centres of the triangle

In der Möbius-Geometrie der Ebene sind Kreise und Geraden (als Kreise durch den unendlich fernen Punkt der Ebene) nicht unterscheidbar. Die übliche Konstruktion der In- und Ankreismittelpunkte eines Dreiecks macht daher möbiusgeometrisch keinen Sinn; erstaunlicherweise lassen sich jedoch diese Zentren eines Dreiecks auch kreisgeometrisch konstruieren. Genauer: es gibt Möbius-Transformationen, die die Ecken des Dreiecks und den unendlich fernen Punkt der Ebene auf die An- und Inkreismittelpunkte abbilden. Eines der beteiligten Symmetriezentren ist Thurstons Symmetriezentrum des idealen Tetraeders im hyperbolischen Raum, dessen Ecken die Ecken des Dreiecks und der unendlich ferne Punkt sind. Dieses Tetraeder, das von den In- und Ankreiszentren geformte Tetraeder und das Tetraeder der vier Symmetriezentren bilden ein desmisches System im umgebenden projektiven Raum. Auf diese Weise ergeben sich Interpretationen der Konfiguration in der Euklidischen Geometrie, der MöbiusGeometrie und der projektiven Geometrie. 
when considering it as a quadrangle in the conformal 2-sphere $S^{2} \cong \mathbb{R}^{2} \cup\{\infty\}$, by using the point at infinity as the fourth point. This has been shown in [4]; here we present an alternative approach.

Perhaps even more surprisingly, it turns out that the obtained configuration of two sets of four points with six interconnecting circles naturally belongs to a spherical geometry: considering each of the two quadrangles as the vertex set of an ideal tetrahedron in hyperbolic space (by thinking of Möbius geometry as the "boundary geometry" of hyperbolic geometry) each can be equipped with Thurston's centre of symmetry [5]; it turns out that the two centres coincide and define an antipodal map that exchanges the in-centre with the point at infinity and the ex-centres with their corresponding vertices. In this way, we obtain a "symmetry breaking phenomenon" for this very simple geometric configuration - this antipodal map defines a natural round metric on the conformal 2-sphere, hence leaving us with the smaller symmetry group of spherical motions.

Clearly both these observations lead to implications for the Euclidean configuration of a triangle with its in- and ex-centres. In particular, we obtain an 8-element abelian symmetry group of the configuration, consisting of Möbius involutions. Of course, triangles in Euclidean geometry have been studied for millenia and it seems unlikely that these implications were not known to some of our illustrious ancestors. However, we have not been able to locate them in the literature so far.

In any case, we hope that the present note can contribute towards a better understanding of the interplay of different geometries: in our case, of

- Euclidean and spherical geometries as subgeometries of Möbius geometry; and of

- Möbius geometry as a boundary geometry of hyperbolic geometry.

Acknowledgements. We would like to express our gratitude to our colleagues D. Calderbank, G. Smith, and T. Soma for fruitful and enjoyable discussions around the subject. Also, we would like to thank the referee for valuable comments on the first version of the paper.

The figures in this text were produced using the excellent geometry sketchpad CaRMetal. Finally, we gratefully acknowledge financial support by JSPS through the first author's long term fellowship grant L-08515, without which we would probably not have started our collaboration on this beautiful topic.

\section{Setting the scene}

We shall start with a brief introduction to the projective model of Möbius geometry used in this article; we hope to provide all essential background for what follows in this way. However, we cannot give a full introduction to Möbius geometry here and the interested reader is referred to Blaschke's classic [1] or to [2] for more comprehensive expositions.

The fundamental idea is to embed the conformal 2-sphere $S^{2} \cong \mathbb{R}^{2} \cup\{\infty\}$ into projective 3 -space $\mathbb{R} P^{3}$ as an absolute quadric, that is, as the projective light cone of a Minkowski $\mathbb{R}^{3,1}$ :

$$
S^{2} \cong P \mathcal{L}^{3} \quad \text { where } \quad \mathcal{L}^{3}=\left\{X=\left.\left(X_{1}, X_{2}, X_{3}, X_{4}\right) \in \mathbb{R}^{3,1}|| X\right|^{2}=0\right\}
$$


denotes the light cone of $\mathbb{R}^{3,1}$ and $|X|^{2}=X_{1}^{2}+X_{2}^{2}+X_{3}^{2}-X_{4}^{2}$ is the quadratic form of the Minkowski inner product $\langle\cdot, \cdot\rangle$ on $\mathbb{R}^{3,1}$. Accordingly, vectors in the light cone are called "light-like", whereas vectors with $|X|^{2}>0$ or $|X|^{2}<0$ are called "space-like" and "time-like", respectively. Thus points in $S^{2} \subset \mathbb{R} P^{3}$ are light-like lines in $\mathbb{R}^{3,1}$.

In this realm, circles can be identified with points "outside" $S^{2} \subset \mathbb{R} P^{3}$ via polarity. In terms of linear algebra, if $c \in \mathbb{R}^{3,1}$ is space-like, i.e., $|c|^{2}>0$, so that $\mathbb{R} c$ is a point "outside" $S^{2} \subset \mathbb{R} P^{3}$, then its orthogonal complement $c^{\perp}$ is a Minkowski subspace that intersects the light cone $\mathcal{L}^{3}$ in a 2-dimensional light cone, that is, the projective plane $\wp$ given by $c^{\perp} \subset \mathbb{R}^{3,1}$ intersects $S^{2}$ in a circle. In terms of projective geometry, $\wp$ is the polar plane of $\mathbb{R} c$ with respect to the absolute quadric $S^{2} \subset \mathbb{R} P^{3}$, that is, the two intersection points of $S^{2}$ with any line $\ell$ in $\mathbb{R} P^{3}$ through $\mathbb{R} c$ separate the points $\mathbb{R} c$ and $\ell \cap \wp$ harmonically. In particular, $\mathbb{R} c$ is the vertex of the cone in $\mathbb{R} P^{3}$ that touches $S^{2}$ along the corresponding circle $\wp \cap S^{2}$. This also explains why the ambient geometry needs to be chosen projective: the vertex of a cylinder, touching along a great circle in $S^{2}$, ends up in the plane at infinity of $\mathbb{R} P^{3}$.

Thus circles in $S^{2} \subset \mathbb{R} P^{3}$ are identified with space-like lines in $\mathbb{R}^{3,1}$, and incidence of a circle and a point is encoded by polarity: a point $\mathbb{R} X$ lies on the circle given by $\mathbb{R} c$ if and only if $X \perp c$.

Normalizing, a circle becomes a pair of antipodal points on the Lorentz sphere

$$
S^{2,1}=\left\{\left.c \in \mathbb{R}^{3,1}|| c\right|^{2}=1\right\},
$$

where each point of the pair (or, equivalently, each ray on the corresponding space-like line) can be thought of as representing an orientation of the circle ${ }^{1)}$. Thus $S^{2,1}$ becomes the space of oriented circles in $S^{2}=\mathbb{R}^{2} \cup\{\infty\}$.

Clearly, any Lorentz transformation

$$
A \in O(3,1)=\{A \in G l(4) \mid\langle A X, A Y\rangle=\langle X, Y\rangle\}
$$

descends to a projective transformation of $\mathbb{R} P^{3}$ that preserves the absolute quadric $S^{2} \subset$ $\mathbb{R} P^{3}$. Hence it acts simultaneously on points and circles in $S^{2}$, preserving the incidence relation. In this way, any Lorentz transformation defines a unique Möbius transformation $\mu: S^{2} \rightarrow S^{2}$, that is, a global conformal transformation of $S^{2}$. Conversely, any Möbius transformation of $S^{2}$ comes from a Lorentz transformation $A$ of $\mathbb{R}^{3,1}$ in this way, $A$ being unique up to sign. In particular, the inversion of $S^{2}$ in a circle given by $c \in S^{2,1}$ comes from a polar reflection

$$
\mathbb{R} X \mapsto \mathbb{R}\{X-2\langle X, c\rangle c\}
$$

of $\mathbb{R} P^{3}$ or, equivalently, from the usual reflection of $\mathbb{R}^{3,1}$ in the Minkowski subspace $c^{\perp}$. Note that the action of this polar reflection on the "outside" of $S^{2} \subset \mathbb{R} P^{3}$ yields the action of the inversion on circles:

$$
S^{2,1} \ni c^{\prime} \mapsto \mp\left(c^{\prime}-2\left\langle c^{\prime}, c\right\rangle c\right) \in S^{2,1}
$$

\footnotetext{
${ }^{1)}$ Classically, Möbius geometry only deals with unoriented circles but, in our quest, orientations will be of assistance.
} 
maps a normalized representative of a circle $c^{\prime}$ to a normalized representative of its image, preserving or reversing orientation of the circle $c$ of inversion.

Finally note that the "inside"

$$
H^{3} \cong\left\{\left.\mathbb{R} Y \in \mathbb{R} P^{3}|| Y\right|^{2}<0\right\}
$$

of $S^{2} \subset \mathbb{R} P^{3}$ can be interpreted as a Klein model of 3-dimensional hyperbolic geometry: the hyperbolic lines are the segments of projective lines that intersect $S^{2}$ in two points, and the hyperbolic motions are the restrictions to the "inside ball" $H^{3} \subset \mathbb{R} P^{3} \backslash S^{2}$ of projective transformations that preserve $S^{2}$ as the infinity boundary of hyperbolic space, that is, of Möbius transformations of $S^{2}$ or, equivalently, Lorentz transformations of $\mathbb{R}^{3,1}$ up to \pm id. In this sense the Möbius geometry of $S^{2}$ becomes the "boundary geometry" of 3-dimensional hyperbolic geometry.

We shall make these ideas more tangible by providing some explicit formulas: consider

$$
\mathbb{R}^{3,1}=\mathbb{R}^{2} \oplus \mathbb{R}^{1,1} \quad \text { with } \quad \mathbb{R}^{1,1}=\operatorname{span}\{o, \infty\},
$$

where $o, \infty$ are light-like with $\langle o, \infty\rangle=-1$, say, $o=\frac{1}{2}(0,0,-1,1)$ and $\infty=(0,0,1,1)$. Now, denoting

$$
\mathcal{Q}^{2}:=\left\{Y \in \mathcal{L}^{3} \mid\langle\infty, Y\rangle=-1\right\},
$$

the map

$$
\mathbb{R}^{2} \ni x \mapsto X=o+x+\frac{|x|^{2}}{2} \infty \in \mathcal{Q}^{2}
$$

is an isometry between 2-dimensional Riemannian manifolds; note that, for $X, Y \in \mathcal{Q}^{2}$,

$$
\langle X, Y\rangle=-\frac{|y-x|^{2}}{2} .
$$

Note the similarity of the formula, using $o$ and $\infty$ as above, with inverse stereographic projection. Indeed, our isometry $\mathbb{R}^{2} \rightarrow \mathcal{Q}^{2}$ is obtained by

- first mapping $\mathbb{R}^{2} \ni x \mapsto \frac{1}{|x|^{2}+1}\left(2 x,|x|^{2}-1\right) \in S^{2} \subset \mathbb{R}^{3}$ by inverse stereographic projection (cf. Fig. 11),

- next embedding $\mathbb{R}^{3} \hookrightarrow \mathbb{R}^{3,1}$ as the affine hyperplane $X_{4}=1$, and

- finally rescaling the light cone vectors to obtain an isometry ${ }^{2}$.

We will henceforth drop the notational distinction between points or circles in $S^{2}$ and their representing vectors in $\mathbb{R}^{3,1}$.

Lines (that is, circles through the point $\infty$ ) and circles can now be written as

$$
\begin{aligned}
& l=n+\langle x, n\rangle \infty, \\
& c=\frac{1}{r}\left(o+m+\frac{|m|^{2}-r^{2}}{2} \infty\right)=(n+\langle x, n\rangle \infty)+\frac{1}{r}\left(o+x+\frac{|x|^{2}}{2} \infty\right),
\end{aligned}
$$

\footnotetext{
${ }^{2)}$ In fact, stereographic projection can be thought of as a simple rescaling $\mathcal{S}^{2} \cong\left\{X \mid X_{4}=1\right\} \ni X \mapsto$ $-\frac{X}{\langle X, \infty\rangle} \in \mathcal{Q}^{2}$ when identifying $\mathcal{Q}^{2} \cong \mathbb{R}^{2}$.
} 
respectively. Here, a circle is given by its radius $r \in \mathbb{R}$ and either by its centre $m \in \mathbb{R}^{2}$ or by a point $x$ on the circle and the normal $n$ at that point so that $m=x+r n$. Note that we take $r<0$ if the circle $c$ is oriented so that its normal $n$ is pointing outwards (cf. [1, $\S \S 12-14])$. The formula for a line is obtained as a limiting case, as $r \rightarrow \pm \infty$.

We also obtain an interpretation for inner products: for $Y \in \mathcal{Q}^{2}$,

$$
\langle l, Y\rangle=\langle y-x, n\rangle=h_{l}
$$

is the (oriented) height of the point $y$ above the line $l$ and

$$
\langle c, Y\rangle=\frac{r^{2}-|y-m|^{2}}{2 r},
$$

confirming that incidence is encoded by orthogonality:

$$
y \in l \Leftrightarrow\langle l, Y\rangle=0 \quad \text { and } \quad y \in c \Leftrightarrow\langle c, Y\rangle=0 .
$$

Also, the curvature $\kappa$ of a circle or a line is given by its scalar product with $\infty$ :

$$
\left.\begin{array}{l}
\langle l, \infty\rangle=0 \\
\langle c, \infty\rangle=\frac{-1}{r}
\end{array}\right\} \Leftrightarrow \kappa=-\langle c, \infty\rangle
$$

reflecting, in particular, that lines are those circles that contain the point at infinity.

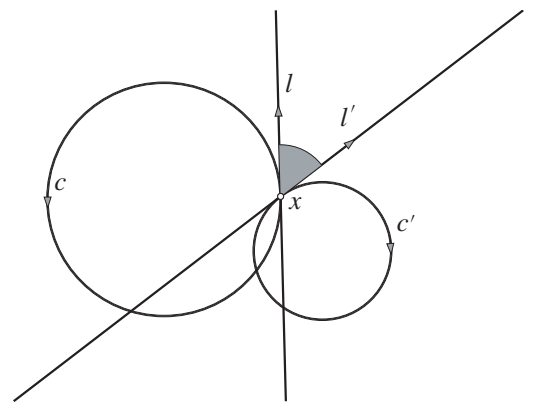

Fig. 1 Intersection angle of two oriented circles: changing the orientation of one circle yields the supplementary angle.

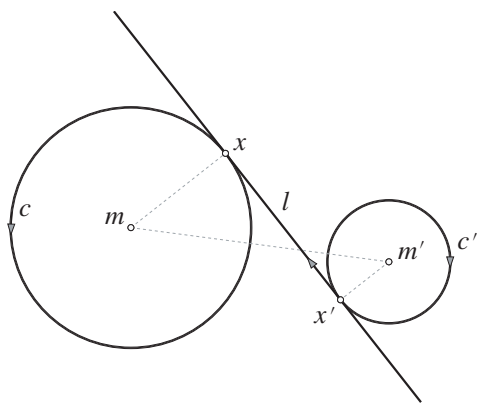

Fig. 2 Tangential distance of two oriented circles: changing the orientation of one circle gives a different tangent line.

Computing the scalar product of two circles $c, c^{\prime} \in S^{2,1}$

$$
\left\langle c, c^{\prime}\right\rangle=-\frac{\left|m^{\prime}-m\right|^{2}-\left(r^{2}+r^{\prime 2}\right)}{2 r r^{\prime}}
$$

in terms of their centres and radii readily reveals that $\left\langle c, c^{\prime}\right\rangle^{2} \leq 1$ if and only if the two circles intersect. Assuming that $c$ and $c^{\prime}$ have a common point $x$, this scalar product

$$
\left\langle c, c^{\prime}\right\rangle=\left\langle l+\frac{1}{r} X, l^{\prime}+\frac{1}{r^{\prime}} X\right\rangle=\left\langle l, l^{\prime}\right\rangle=\left\langle n, n^{\prime}\right\rangle=\cos \angle\left(c, c^{\prime}\right)
$$


yields their oriented angle of intersection; in particular, two circles $c, c^{\prime}$ intersect orthogonally if and only if $c \perp c^{\prime}$ and they are in oriented (or orientation reversing) contact if and only if $\left\langle c, c^{\prime}\right\rangle=1$ (or $\left\langle c, c^{\prime}\right\rangle=-1$ ). Note that, in case the two circles have a common (oriented) tangent line $l$, their scalar product can be interpreted in terms of their tangential distance:

$$
\left\langle c, c^{\prime}\right\rangle=\left\langle l+\frac{1}{r} X, l+\frac{1}{r^{\prime}} X^{\prime}\right\rangle=1-\frac{\left|x^{\prime}-x\right|^{2}}{2 r r^{\prime}} .
$$

As any two lines have the point at infinity in common, their scalar product gives the angle of intersection, or it is 1 (or -1 ) for parallel (or anti-parallel) lines that touch at $\infty$.

Finally, we recall the notion of a "circle pencil", that is, the configuration of circles that belong to a line in $\mathbb{R} P^{3}$ or, equivalently, to a 2-plane $\pi \subset \mathbb{R}^{3,1}$. These come in three different flavours.

- Elliptic circle pencils, where the line does not meet $S^{2}$, i.e., where $\pi$ is a Euclidean plane: the circles in $\pi$ are those that contain the two points given by the null lines in $\pi^{\perp}$.

- Hyperbolic circle pencils, where the line intersects $S^{2}$ transversally, i.e., where $\pi$ is a Minkowski plane: we get those circles that are orthogonal to all circles in $\pi^{\perp}$ if $\infty \in \pi$ then we obtain concentric circles with their common centre given by the second null line in $\pi$.

- Parabolic circle pencils, where the line touches $S^{2}$, i.e., where $\pi$ has a degenerate metric: here, the null line in $\pi$ defines a point $x$ in which all the circles of the pencil touch - note that, in this case, $\pi \cap \pi^{\perp}=\operatorname{span}\{X\}$ and $\pi+\pi^{\perp}$ yields the complex of all circles containing $x$.

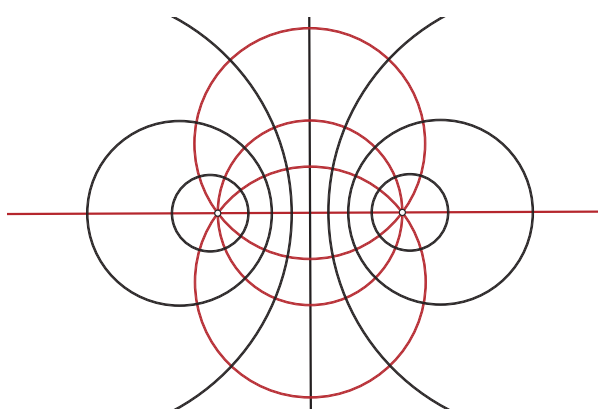

Fig. 3 Orthogonally intersecting hyperbolic (black, containing two point circles) and elliptic (red) circle pencils.

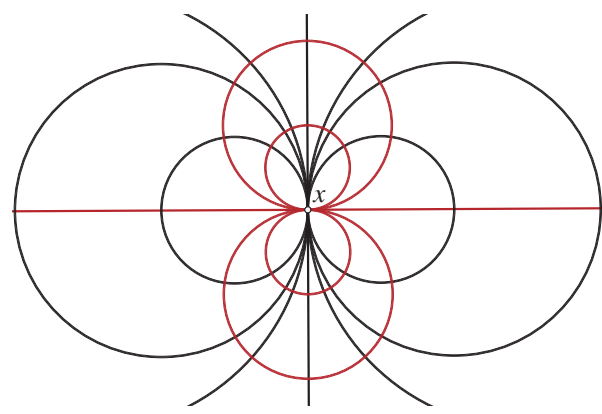

Fig. 4 Two parabolic circle pencils (or, "contact elements") that intersect orthogonally at the point $x$.

\section{The in- and ex-centres of a Euclidean triangle}

Now fix a non-degenerate triangle $(A, B, C)$ in $\mathbb{R}^{2} \cong \mathcal{Q}^{2}$ : thus $A, B, C \in \mathcal{Q}^{2}$ and $(A, B, C, \infty)$ form a basis of $\mathbb{R}^{3,1}$. Let $a, b$ and $c$ denote the sides of the triangle, as usual opposite to $A, B$ and $C$, respectively: thus $a \in S^{2,1}$ is the circle through $B, C$ and 
$\infty$, etc.; we fix the orientations of $a, b$ and $c$ so that the corresponding heights are positive:

$$
h_{a}=\langle a, A\rangle, \quad h_{b}=\langle b, B\rangle, \quad h_{c}=\langle c, C\rangle>0 \text {. }
$$

Note that $(a, b),(b, c)$ and $(c, a)$ each span an elliptic circle pencil with $(\infty, C),(\infty, A)$ and $(\infty, B)$ as their respective intersection points; on the other hand, $a, b$ and $c$ have only one common intersection point $\infty$, reflecting the linear independence of $a, b$ and $c$.

We now seek the in-circle $i$ of the triangle, that is, a circle $i \in S^{2,1}$ that has oriented contact with all three sides of the triangle. In particular,

$$
1=\langle i, a\rangle=\langle i, b\rangle=\langle i, c\rangle \quad \Rightarrow \quad i \perp a-b, b-c, c-a,
$$

that is, we seek $i$ in the circle pencil given by the angle bisectors of the triangle. To see this, first note that

$$
b-c \perp \infty, A \quad \text { and } \quad\langle b-c, b\rangle+\langle b-c, c\rangle=0
$$

showing that $b-c$ is a line through $A$ so that its angles with $b$ and $c$ add up to $\pi$, that is, $b-c$ bisects the (inner) angle of the triangle at $A$; similarly $c-a$ and $a-b$ are the (inner) angle bisectors of the triangle at $B$ and $C$, respectively. Secondly, dim $\operatorname{span}\{a-b, b-c, c-a\}=$ 2 as $a, b, c$ are linearly independent so that

$$
\operatorname{span}\{a-b, b-c, c-a\}=: \eta
$$

defines an elliptic or parabolic circle pencil since $\infty \in \eta^{\perp}$. From Euclidean geometry we know that $\eta$ defines an elliptic circle pencil with $\infty$ and the in-centre as its two intersection points; we shall confirm this using our Möbius geometric setting.

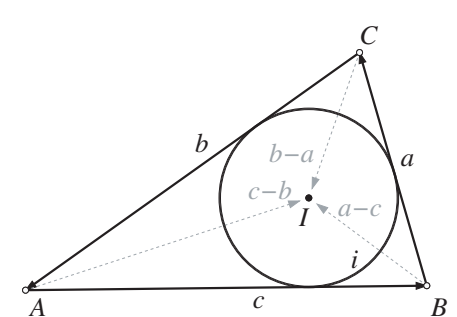

Fig. 5 The in-centre construction of a triangle: the orientations of the sides pick the inner angle bisectors.

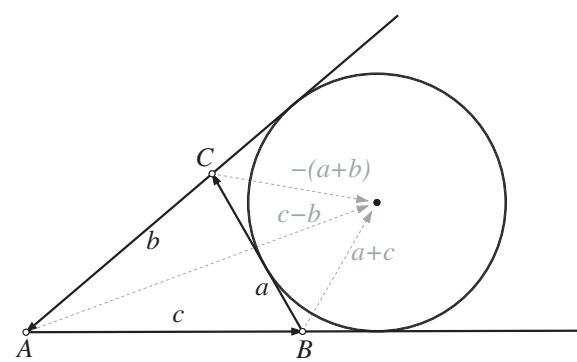

Fig. 6 The ex-centre construction for a triangle: a swap of orientation of one side changes two of the angle bisectors.

To this end we first derive a formula for the circum-radius $R$ of our triangle: let $l_{a}, l_{b}$ and $l_{c}$ denote the side lengths of the triangle and $F$ its area; then

$$
R=\frac{l_{a} l_{b} l_{c}}{4 F}=\frac{2 F l_{c}}{2} \frac{l_{a}}{2 F} \frac{l_{b}}{2 F}=\frac{l_{c}^{2} h_{c}}{2 h_{a} h_{b}}=-\frac{\langle A, B\rangle\langle c, C\rangle}{\langle a, A\rangle\langle b, B\rangle} .
$$


Lemma 3.1. The circum-radius $R$ of the triangle $(A, B, C) \subset \mathcal{Q}^{2}$ satisfies

$$
\left\langle\frac{A}{\langle a, A\rangle}, \frac{B}{\langle b, B\rangle}\right\rangle=\left\langle\frac{C}{\langle c, C\rangle}, R \infty\right\rangle \text {. }
$$

Clearly two more formulas are obtained by permutation. As a consequence,

$$
\left|\frac{A}{\langle a, A\rangle}+\frac{B}{\langle b, B\rangle}+\frac{C}{\langle c, C\rangle}\right|^{2}=2 R\left\langle\infty, \frac{A}{\langle a, A\rangle}+\frac{B}{\langle b, B\rangle}+\frac{C}{\langle c, C\rangle}\right\rangle ;
$$

further,

$$
\left\langle\infty, \frac{A}{\langle a, A\rangle}+\frac{B}{\langle b, B\rangle}+\frac{C}{\langle c, C\rangle}\right\rangle=-\left\{\frac{1}{h_{a}}+\frac{1}{h_{b}}+\frac{1}{h_{c}}\right\}=-\frac{l_{a}+l_{b}+l_{c}}{2 F}=-\frac{1}{r}
$$

yields the in-radius. Hence

$$
\left|\frac{A}{\langle a, A\rangle}+\frac{B}{\langle b, B\rangle}+\frac{C}{\langle c, C\rangle}-\lambda \infty\right|^{2}=2 \frac{\lambda-R}{r}= \begin{cases}0 & \text { if } \lambda=R \\ 1 & \text { if } \lambda=R+\frac{r}{2}\end{cases}
$$

providing explicit formulas for the in-centre and in-circle:

Lemma 3.2. The in-centre I and in-circle i of a triangle $(A, B, C) \subset \mathcal{Q}^{2}$ are given by $\left.{ }^{3}\right)$

$$
I=\frac{A}{\langle a, A\rangle}+\frac{B}{\langle b, B\rangle}+\frac{C}{\langle c, C\rangle}-R \infty \quad \text { and } \quad i=I-\frac{r}{2} \infty .
$$

Proof. Clearly, $\langle i, a\rangle=\langle i, b\rangle=\langle i, c\rangle=1$ establishing $i \in S^{2,1}$ as the in-circle of the triangle. Moreover,

$$
I, \infty \in \eta^{\perp}=\{a-b, b-c, c-a\}^{\perp}
$$

showing that $\eta$ defines an elliptic circle pencil ${ }^{4)}$ consisting of the lines through $I$ and intersecting the in-circle $i$ perpendicularly since

$$
i \in \operatorname{span}\{\infty, I\}=\eta^{\perp} .
$$

Consequently, the (second) intersection point $I$ of the (inner) angle bisectors $a-b, b-c$ and $c-a$ of the triangle is, not surprisingly, the in-centre of the triangle ${ }^{5)}$.

The ex-circles and ex-centres of the triangle can now be constructed in a very similar way, by simply changing our mind about the orientation of one of the edges: to construct the ex-circle opposite the point $A$, say, we seek a circle

$$
j_{A} \perp \operatorname{span}\{a+b, a+c, b-c\}=: \epsilon_{A} ;
$$

again, we obtain its centre $J_{a}$ as the second point of intersection of the circles in the elliptic circle pencil given by $\epsilon_{A}$ and the ex-circle itself as an appropriate circle in the orthogonal hyperbolic pencil $\epsilon_{A}^{\perp}=\operatorname{span}\left\{\infty, J_{a}\right\}$. Thus, similarly to the proof of Lemma 3.2 we obtain:

\footnotetext{
${ }^{3)}$ Scaling $I$ by the in-radius provides the representative $r I \in \mathcal{Q}^{2}$.

${ }^{4)}$ Here we use that $(A, B, C)$ is a non-degenerate triangle, so that $R<\infty$.

5) Alternatively, we could argue that $\infty-2\langle\infty, i\rangle i=\infty+\frac{2}{r}\left(I-\frac{r}{2} \infty\right) \| I$, that is, the inversion in $i$ interchanges $I$ and $\infty$ and, hence, $I$ is the centre of $i$.
} 
Lemma 3.3. The ex-centre $J_{a}$ and ex-circle $j_{A}$ opposite A of a triangle $(A, B, C) \subset \mathcal{Q}^{2}$ are given by

$$
J_{a}=-\frac{A}{\langle a, A\rangle}+\frac{B}{\langle b, B\rangle}+\frac{C}{\langle c, C\rangle}+R \infty \text { and } j_{A}=J_{a}-\frac{r_{A}}{2} \infty,
$$

where $r_{A}$ denotes the corresponding ex-radius,

$$
\frac{1}{r_{A}}=-\left\langle\infty,-\frac{A}{\langle a, A\rangle}+\frac{B}{\langle b, B\rangle}+\frac{C}{\langle c, C\rangle}\right\rangle .
$$

The ex-centres $J_{b}, J_{c}$ and corresponding ex-circles $j_{B}, j_{C}$ are given by similar formulas.

Note that changing orientation of two or all three edges of our triangle does not provide new circles via the described construction: we just obtain the ex- and in-circles with opposite orientations.

Also note that, as a simple consequence, we obtain the well-known formula relating the in- and ex-radii of a triangle:

$$
\frac{1}{r_{A}}+\frac{1}{r_{B}}+\frac{1}{r_{C}}=\frac{1}{r}
$$

\section{The conformal dual of four points}

We shall now pursue a geometric construction that explains the striking symmetry that we observe in the formulas for the in- and ex-centres of a triangle given in Lemmas 3.2 and 3.3.

In the previous section we have investigated the geometry of a Euclidean triangle - the geometry of a triangle in the presence of a distinguished point $\infty$, which also provided a distinction of lines as those circles containing $\infty$. We shall now drop this distinction of a special point and consider the geometry of a non-concircular quadrangle $\left.{ }^{6}\right)(A, B, C, D) \in$ $S^{2}$ without distinguishing one of the four points ${ }^{7)}$.

Thus let $(A, B, C, D) \subset S^{2}$ be a quadrangle in the conformal 2-sphere, and let

$$
a, b, c, d \in S^{2,1}=\left\{\left.Y \in \mathbb{R}^{3,1}|| Y\right|^{2}=1\right\}
$$

represent the circles containing all but one of the points of the quadrangle, that is, $a$ is the circle through $B, C, D$, etc. We choose the orientation of each circle so that its normal points into the component of its complement in $S^{2}$ which contains the corresponding point $^{8)}$. Finally, we fix homogeneous coordinates ${ }^{9)}$,

$$
A, B, C, D \in \mathcal{L}^{3}=\left\{\left.Y \in \mathbb{R}^{3,1}|| Y\right|^{2}=0\right\}
$$

\footnotetext{
6) As any two triangles are Möbius equivalent, a quadrangle is the simplest geometric configuration of interest in Möbius geometry.

${ }^{7)}$ For illustrative purposes as well as for proofs it will, however, often be convenient to consider one of the points, say $D$, as the point at infinity of a Euclidean plane. We shall primarily be interested in geometric properties of the configurations that remain invariant under the full symmetry group of Möbius transformations though.

${ }^{8)}$ Reversing orientation of two of these circles would not change much, in particular, the alternate segment theorem (Lemma 4.3) would not be affected: we shall come back to this point later.

${ }^{9}$ Remember that we do not distinguish notationally between a point in $S^{2} \subset \mathbb{R} P^{3}$ and a homogeneous coordinate vector of this point in $\mathcal{L}^{3} \subset \mathbb{R}^{3,1}$.
} 
so that $(a, b, c, d)$ and $(A, B, C, D)$ form dual bases of $\mathbb{R}^{3,1}: A \perp b, c, d$ since the circles $b, c, d$ intersect in the point $A$, etc. Hence, by a suitable choice of homogeneous coordinates, we can achieve that

$$
1=\langle a, A\rangle=\langle b, B\rangle=\langle c, C\rangle=\langle d, D\rangle
$$

while all other pairings using the scalar product vanish. Note that, in general, $A, B, C$, $D \notin \mathcal{Q}^{2}$ as they are now differently normalized.

Observe that by considering $D$ as the point at infinity of $S^{2} \cong \mathbb{R}^{2} \cup\{\infty\}$ we make contact with the Euclidean triangle geometry of the previous section:

- the homogeneous coordinates of the points $A, B$ and $C$ are now rescaled, $A \mapsto \frac{A}{\langle a, A\rangle}$, etc.;

- the circle $d$ becomes the circum-circle of the triangle $(A, B, C)$, oriented so that its normal points outwards, that is, its radius becomes $-|R|<0$; and

- $D=\frac{\infty}{\langle d, \infty\rangle}=|R| \infty$.

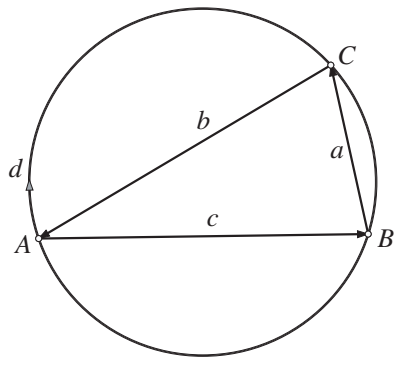

Fig. 7 Orientations of four circles through three of four points: the point not on the circle always lies on the side the positively oriented normal points into.

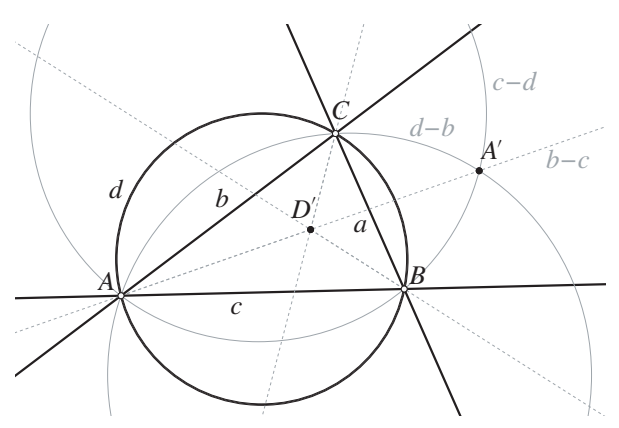

Fig. 8 A Möbius geometric in-centre construction: here, $A$ takes the role of the point at infinity of a Euclidean plane to obtain the point $A^{\prime}$.

Hence we obtain now a completely symmetric set of formulas for the ex-centres $A^{\prime}, B^{\prime}$, $C^{\prime}$ and the in-centre $D^{\prime}$ of the triangle $(A, B, C) \subset \mathcal{Q}^{2}$ from Lemmas 3.3 and 3.2:

$$
\begin{aligned}
A^{\prime} & =\frac{1}{2}\{-A+B+C+D\}, \\
B^{\prime} & =\frac{1}{2}\{A-B+C+D\}, \\
C^{\prime} & =\frac{1}{2}\{A+B-C+D\}, \\
D^{\prime} & =\frac{1}{2}\{A+B+C-D\} .
\end{aligned}
$$

From this symmetry it becomes clear that the ex-centres can alternatively be obtained by an in-centre construction when the appropriate point is considered as the point at infinity: 
for example, $A^{\prime}$ can be constructed as the in-centre of the triangle $B, C, D$ when $A$ is considered as the point at infinity.

A Möbius geometric formulation of this fact reveals a more symmetric picture of the situation, cf. [4, Lemma 1.5]:

Theorem 4.1. The ex- and in-centres of a triangle in Euclidean space can be constructed in a symmetric, Möbius geometric way from the vertices of the triangle and the point at infinity.

Proof. Fix one point of the quadrangle $(A, B, C, D) \subset S^{2}$, say $A$; we wish to construct $A^{\prime}$ by Möbius geometric means. Thus let $i_{A}$ denote the (unique) circle in oriented contact with the three circles $b, c, d$ - then $A^{\prime}$ is obtained by inverting $A$ in the circle $i_{A}$.

Equivalently, $A^{\prime}$ can be obtained as the second intersection point of the elliptic circle pencil spanned by the circles $b-c, c-d$ and $d-b$ through $A$ and bisecting the angles between $b$ and $c$ at $D, c$ and $d$ at $B$, and $d$ and $b$ at $C$, respectively.

This latter construction characterizes the quadrangle $\left(A^{\prime}, B^{\prime}, C^{\prime}, D^{\prime}\right)$ as the "conformal dual" of $(A, B, C, D)$ in the sense of [4, Def. 1.2]:

Definition 4.2. $\left(A^{\prime}, B^{\prime}, C^{\prime}, D^{\prime}\right)$ is called the conformal dual of the non-circular quadrangle $(A, B, C, D)$.

Below, we shall provide a new proof for [4, Thm. 1.4], confirming that this yields a sensible notion of "duality", that is, that

$$
\left(A^{\prime \prime}, B^{\prime \prime}, C^{\prime \prime}, D^{\prime \prime}\right)=(A, B, C, D) .
$$

First note that the dual quadrangle is, as the original $(A, B, C, D)$, in general position, that

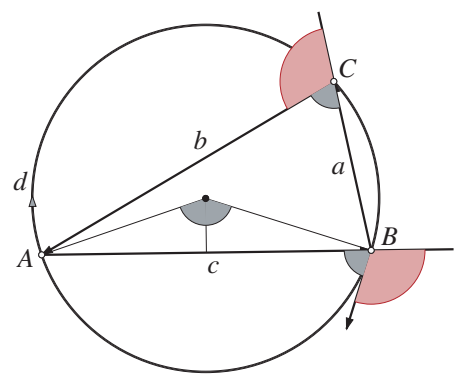

Fig. 9 The alternate segment theorem: the angle between the secant and the tangent to the circum-circle equals the inscribed angle in the alternate segment of the circle; hence $\langle a, b\rangle=\langle c, d\rangle$.

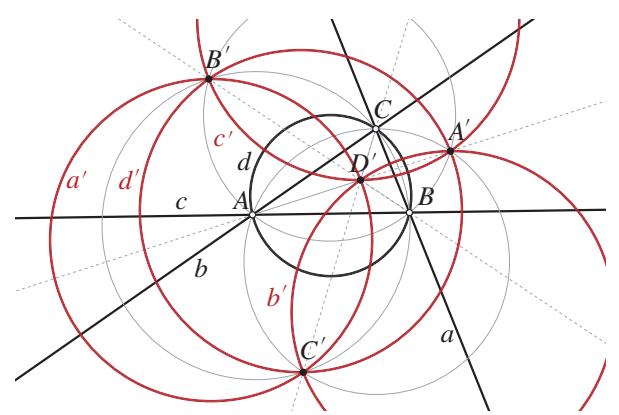

Fig. 10 The "conformal dual" of a quadrangle: the points $A^{\prime}, B^{\prime}, C^{\prime}$ and $D^{\prime}$ are obtained by Möbius geometric in-circle constructions, considering $A, B, C$ and $D$ as the point at infinity, respectively. 
is, $\left(A^{\prime}, B^{\prime}, C^{\prime}, D^{\prime}\right)$ is a basis of $\mathbb{R}^{3,1}$; further,

$$
\begin{aligned}
a^{\prime} & :=\frac{1}{2}\{-a+b+c+d\}, \\
b^{\prime} & :=\frac{1}{2}\{a-b+c+d\}, \\
c^{\prime} & :=\frac{1}{2}\{a+b-c+d\}, \\
d^{\prime} & :=\frac{1}{2}\{a+b+c-d\}
\end{aligned}
$$

clearly define its dual basis,

$$
\left\langle x^{\prime}, Y^{\prime}\right\rangle=\delta_{x^{\prime} Y^{\prime}}:= \begin{cases}1 & \text { if } Y^{\prime}=X^{\prime} \\ 0 & \text { otherwise }\end{cases}
$$

Moreover, $a^{\prime}, b^{\prime}, c^{\prime}, d^{\prime} \in S^{2,1}$ define oriented circles:

$$
\left|a^{\prime}\right|^{2}=1+\frac{(\langle c, d\rangle-\langle a, b\rangle)+(\langle b, d\rangle-\langle a, c\rangle)+(\langle b, c\rangle-\langle a, d\rangle)}{2}=1
$$

by the following lemma, complementing the formulas

$$
\langle A, B\rangle=\langle C, D\rangle
$$

as obtained from Lemma 3.1:

Lemma 4.3. Pairs of opposite circular edges of a quadrangle $(A, B, C, D) \subset S^{2}$ intersect at equal angles:

$$
\langle a, b\rangle=\langle c, d\rangle
$$

Proof. Considering one of the points, say $D$, as the point at infinity of $S^{2} \cong \mathbb{R}^{2} \cup\{\infty\}$, this lemma becomes the alternate segment theorem and is a direct consequence of the generalized Thales theorem (or, "inscribed angle theorem"): the inscribed angle over a secant is half the central angle which, in turn, is the angle that the secant makes with the tangent to the circum-circle; the angle between $c$ and $d$ is the supplementary angle of the latter whereas the angle between $a$ and $b$ is the supplementary angle of the former.

Now, as $\left(b^{\prime}-c^{\prime}\right)+(b-c)=0$, etc., we learn that the original quadrangle is constructed from its dual in the same way the dual is from the original, cf. [4, Thm. 1.4]:

$$
A, A^{\prime} \perp b^{\prime}-c^{\prime}, c^{\prime}-d^{\prime}, d^{\prime}-b^{\prime} .
$$

Theorem 4.4. The notion of "conformal duality" is symmetric,

$$
\left(A^{\prime \prime}, B^{\prime \prime}, C^{\prime \prime}, D^{\prime \prime}\right)=(A, B, C, D) .
$$




\section{The conformal centre of four points}

We shall now give another description of the relation between a quadrangle and its dual, that is, between a Euclidean triangle and its in- and ex-centres, that is palpably symmetric.

Definition 5.1. We define the conformal centre $Z$ of our quadrangle $(A, B, C, D) \subset S^{2}$ by:

$$
Z:=\frac{1}{2}(A+B+C+D)
$$

Recall that we chose homogeneous coordinates $A, B, C, D \in \mathcal{L}^{3}$ of the four vertices of our quadrangle that were uniquely determined by the geometry of the quadrangle so that $Z$ is well defined; also recall that, applying Lemma 3.1 to these vectors, we obtain $\langle A, B\rangle=$ $\langle C, D\rangle$ while Lemma 4.3 provided us with $\langle a, b\rangle=\langle c, d\rangle$. Hence $|Z|^{2}=2\langle Z, A\rangle$ and similarly for $B, C$ and $D$. Writing $Z$ in terms of the dual basis $(a, b, c, d)$ now yields

$$
Z=\langle Z, A\rangle a+\langle Z, B\rangle b+\langle Z, C\rangle c+\langle Z, D\rangle d=\frac{1}{2}|Z|^{2}\{a+b+c+d\}
$$

implying ${ }^{10)}$, in particular, that $|Z|^{2} \neq 0$ - so that reflection in $Z^{\perp}$ yields an interesting Möbius involution of $S^{2}$ :

Lemma 5.2. The Möbius involution

$$
\mu: S^{2} \rightarrow S^{2}, \quad X \mapsto-\left\{X-2 \frac{\langle X, Z\rangle}{|Z|^{2}} Z\right\}
$$

exchanges the quadrangle $(A, B, C, D)$ and its conformal dual $\left(A^{\prime}, B^{\prime}, C^{\prime}, D^{\prime}\right)$ :

$$
\mu(A)=Z-A=A^{\prime} \quad \text { and } \quad \mu(a)=\frac{Z}{|Z|^{2}}-a=a^{\prime}, \quad \text { etc. }
$$

Observe that

$$
Z=\frac{1}{2}(A+B+C+D)=A+A^{\prime}=\frac{1}{2}\left(A^{\prime}+B^{\prime}+C^{\prime}+D^{\prime}\right)
$$

so that $Z=Z^{\prime}$ is also the conformal centre of the dual quadrangle. Thus we obtain an alternative proof of Theorem 4.4. Also, as $\mu$ is orientation reversing, we obtain a new proof of $[4$, Thm. 1.8]:

Corollary 5.3. The cross ratios of conformally dual quadrangles are complex conjugates of each other.

In order to understand the geometry of $Z$ and the corresponding involution $\mu$ better, consider the circle pencils

$$
\pi^{ \pm}:=\operatorname{span}\{a \pm b, c \pm d\}
$$

\footnotetext{
${ }^{10)}$ We could also argue here that $Z$ is a convex combination of vectors in one component of $\mathcal{L}^{3}$, implying that $|Z|^{2}<0$. However, we shall provide another, more geometric proof of this fact below, after clarifying the geometric setup.
} 
By Lemma 4.3, $a \pm b \perp c \mp d$ so that the circle pencils $\pi^{+}$and $\pi^{-}$are orthogonal. We wish to show that $\pi^{-}$defines an elliptic circle pencil: consider $D=\infty$ as the point at infinity; then $d$ is the circum-circle of the triangle $(A, B, C)$ and $c-d$ is a circle containing $A$ and $B$; on the other hand, $a-b$ is the inner angle bisector of the triangle at $C$, hence intersects the side $c$ between $A$ and $B$, that is, in the inside of the circle $c-d$. Consequently, $a-b$ and $c-d$ intersect in two points and, therefore, define an elliptic circle pencil.

Now consider the projective transformation on $\mathbb{R} P^{3}$ given by rotation by $\theta$ in the (Euclidean) plane $\pi^{-}$:

- on the one hand, this yields a Möbius transformation of $S^{2} \subset \mathbb{R} P^{3}$ that fixes each circle in the pencil $\pi^{+}$- if we regard one of the two point circles in the pencil as the point at infinity we obtain a rotation around the other;

- on the other hand, if we consider the interior of $S^{2} \subset \mathbb{R} P^{3}$ as the Klein model of a hyperbolic 3-space, this yields a hyperbolic rotation with the hyperbolic line defined by $\pi^{+}$as its axis.

If we choose, in particular, the rotation angle $\theta=\pi$ then this transformation

$$
\begin{aligned}
& a=\frac{a+b}{2}+\frac{a-b}{2} \mapsto \frac{a+b}{2}-\frac{a-b}{2}=b, \\
& b=\frac{a+b}{2}-\frac{a-b}{2} \mapsto \frac{a+b}{2}+\frac{a-b}{2}=a, \\
& c=\frac{c+d}{2}+\frac{c-d}{2} \mapsto \frac{c+d}{2}-\frac{c-d}{2}=d, \\
& d=\frac{c+d}{2}-\frac{c-d}{2} \mapsto \frac{c+d}{2}+\frac{c-d}{2}=c ;
\end{aligned}
$$

hence it acts as a permutation on the quadrangle $(A, B, C, D)$,

$$
(A, B, C, D) \mapsto(B, A, D, C),
$$

and thus provides one of the three non-trivial symmetries of the ideal tetrahedron in hyperbolic 3-space with our quadrangle as its ideal vertices, cf. [5, Ch. 4]. By symmetry, we obtain three such hyperbolic rotations, $\rho_{a b}, \rho_{a c}$ and $\rho_{a d}$, with axes given by

$$
\operatorname{span}\{a+b, c+d\}, \quad \operatorname{span}\{a+c, b+d\} \quad \text { and } \operatorname{span}\{a+d, b+c\},
$$

respectively; these axes intersect orthogonally, e.g., $\langle(a+b)-(c+d),(a+c)-(b+d)\rangle=0$ by Lemma 4.3, so that the $\varrho_{x y}$ form a Klein four-group. Moreover, the axes intersect in a unique point in hyperbolic space,

$$
\mathbb{R}(a+b+c+d)=\mathbb{R} Z .
$$

Theorem 5.4. Considering the interior of $S^{2} \subset \mathbb{R} P^{3}$ as a Klein model of hyperbolic space, the conformal centre $Z$ of a quadrangle $(A, B, C, D) \subset S^{2}$ is Thurston's hyperbolic centre of symmetry of the ideal tetrahedron with ideal vertices $A, B, C$ and $D$. 
Apart from providing another geometric interpretation for the conformal centre of a quadrangle in $S^{2}$, this also yields further insight into the geometry of the involution $\mu$.

First note that from

$$
2\langle A, Z\rangle=|Z|^{2} \quad \text { and } \quad 2\left\langle A^{\prime}, Z\right\rangle=2\langle Z-A, Z\rangle=|Z|^{2}
$$

and similarly for $B, C$ and $D$, we learn that

$$
(A, B, C, D),\left(A^{\prime}, B^{\prime}, C^{\prime}, D^{\prime}\right) \subset \mathcal{S}^{2}:=\left\{Y \in \mathcal{L}^{3} \mid\left\langle Y, \frac{2 Z}{|Z|^{2}}\right\rangle=1\right\},
$$

that is, our quadrangles lie in a round 2-sphere with curvature $\frac{-4}{|Z|^{2}}$, see [2, Sect. 1.4]. The point $\frac{1}{2} Z$ is the centre of $\mathcal{S}^{2}$ in its (Euclidean) affine hyperplane in $\mathbb{R}^{3,1}$ and $\mu$ becomes the antipodal map of $\mathcal{S}^{2}$. Hence we obtain the following result for Euclidean triangles:

Theorem 5.5. Given a quadrangle $(A, B, C, D) \in \mathcal{S}^{2}$ with centre of mass $\frac{A+B+C+D}{4}$ at the centre of $\mathcal{S}^{2}$, stereographic projection from $D$ maps the antipodal quadrangle $\left(A^{\prime}, B^{\prime}, C^{\prime}, D^{\prime}\right)$ to the ex-and in-centres of the triangle obtained by stereographic projection of $(A, B, C)$.

Conversely, given a triangle $(A, B, C) \subset \mathcal{Q}^{2}$ in the Euclidean plane, a suitable (inverse) stereographic projection to $\mathcal{S}^{2}$ maps

- its in-centre to the antipodal point of the centre $\infty$ of the stereographic projection and

- its ex-centres $A^{\prime}, B^{\prime}$ and $C^{\prime}$ to the antipodal points of the images of $A, B$ and $C$, respectively.

To make the latter claim of this theorem more tangible we specify the "suitable" stereographic projection, by specifying the equator $e \in S^{2,1}$ of $\mathcal{S}^{2}$ in the Euclidean plane - the stereographic projection will then be from the north pole of $\mathcal{S}^{2}$ onto the equator plane.

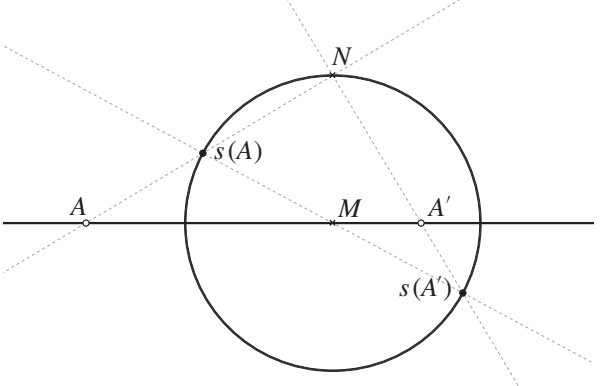

Fig. 11 The inverse stereographic projections $s(A)$ and $s\left(A^{\prime}\right)$ are antipodal, $s(A)+s\left(A^{\prime}\right)=M$, iff $\left\langle A-M, A^{\prime}-M\right\rangle+|N-M|^{2}=0$.

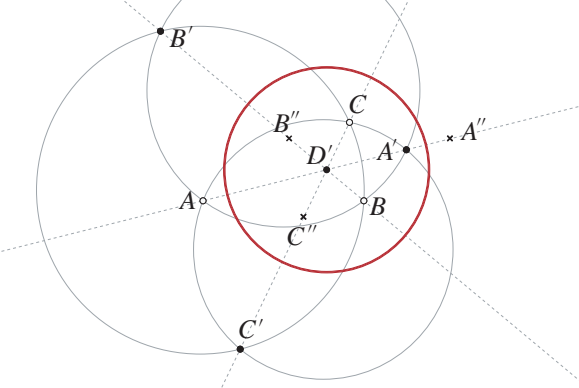

Fig. 12 Inverse stereographic projection of the pairs $\left(A, A^{\prime}\right)$, etc., yields antipodal points on the sphere: inversion of $A^{\prime}$ in the equator gives a pair $\left(A, A^{\prime \prime}\right)$ that is symmetric with respect to the centre $D^{\prime}$ of the equator. 
As the in-centre will be obtained by stereographic projection of the south pole, the equator of $\mathcal{S}^{2}$ has to be centred at the in-centre of the triangle. Further, for a triangle vertex and its corresponding ex-centre to be projections of antipodal points, the geometric mean of their respective distances to the in-centre has to be the radius of the equator - in particular, the three geometric means associated to the three vertices of the triangle have to be the same.

In order to verify this fact, we use the notations and normalizations of this section, that is, $(A, B, C, D)$ and $(a, b, c, d)$ are dual bases of $\mathbb{R}^{3,1}$, and we take $\infty=-\frac{1}{R} D$ with the circum-radius $R<0$ to represent the point at infinity. Denoting by $l_{A, D^{\prime}}$ and $l_{A^{\prime} D^{\prime}}$ the respective distances of $A$ and the ex-centre $A^{\prime}$ to the in-centre $D^{\prime}$, we obtain

$$
l_{A, D^{\prime}}^{2} l_{A^{\prime} D^{\prime}}^{2}=4\left\langle\frac{A}{\langle A, \infty\rangle}, \frac{D^{\prime}}{\left\langle D^{\prime}, \infty\right\rangle}\right\rangle\left\langle\frac{A^{\prime}}{\left\langle A^{\prime}, \infty\right\rangle}, \frac{D^{\prime}}{\left\langle D^{\prime}, \infty\right\rangle}\right\rangle=\frac{4 R^{4}}{\left\langle D^{\prime}, D\right\rangle^{2}}
$$

since $\langle A, B\rangle=\langle C, D\rangle$, etc., by Lemma 3.1. Hence, the radius of the equator can be chosen consistently. Indeed, the antipodal map $\mu$ can be decomposed into three inversions in orthogonal great circles of $\mathcal{S}^{2}$ - choosing two of them as reflections in orthogonal lines through the in-centre $D^{\prime}$, the third becomes the inversion in the sought equator $e$ : hence

$$
e=\frac{D^{\prime}-D}{\left|D^{\prime}-D\right|}=\frac{D^{\prime}-D}{\sqrt{-2\left\langle D^{\prime}, D\right\rangle}}
$$

as $D^{\prime}-D \perp \eta=\operatorname{span}\{a-b, b-c, c-a\}$ and $D^{\prime}-D \perp D^{\prime}+D=Z$; note that, indeed, the radius of $e$ is

$$
-\frac{1}{\langle\infty, e\rangle}=-R \frac{\sqrt{-2\left\langle D^{\prime}, D\right\rangle}}{-\left\langle D^{\prime}, D\right\rangle}=-R \sqrt{-\frac{2}{\left\langle D^{\prime}, D\right\rangle}}=-R \sqrt{\frac{4}{R\langle i, \infty\rangle}}=2 \sqrt{-r R}
$$

where, from Lemma 3.2, $i=2 D^{\prime} \bmod D$ is the in-circle of the triangle and $r>0$ its radius.

\section{The Möbius symmetries of dual quadrangles}

We have already seen four symmetries of a dual pair of quadrangles, that is in Euclidean terms, of the two quadrangles formed by the point at infinity and a triangle and by its inand ex-centres, respectively:

- the antipodal map $\mu$ which interchanges the two quadrangles, and

- the three hyperbolic rotations $\rho_{a b}$, etc., which simultaneously interchange pairs of points in both quadrangles - note that these rotations act on the dual quadrangle in the same way as they do on the original since they commute with the antipodal map.

As $\mu$ commutes with each rotation $\varrho_{x y}$, these transformations generate an 8-element symmetry group of the configuration. To identify the remaining symmetries in this group, we introduce

$$
Z_{a d}:=\frac{1}{2}\{A-B-C+D\}=A-D^{\prime}=D-A^{\prime}
$$


Observe that $Z_{a d} \perp Z$ so that, in particular, $\left|Z_{a d}\right|^{2}>0$ and $Z_{a d}$ defines a circle. Note that this circle becomes a great circle in the spherical geometry defined by $Z$ as the centre of $\mathcal{S}^{2}$ since it lies in the plane of the circle $Z_{a d}$. Consequently, inversion $\mu_{a d}$ in the circle $Z_{a d}$ commutes with the antipodal map $\mu$.

Clearly, since $Z \| a+b+c+d$,

$$
Z_{a d} \perp a-d, b-c, a+b+c+d
$$

so that $\mu_{a d}$ fixes the angle bisectors $a-d$ and $b-c$ as well as $Z$; consequently, using Lemma 4.3, we conclude that

$$
Z_{a d} \| \frac{1}{2}(a-b-c+d)
$$

and $\mu_{a d}$ exchanges the circles given by $a+d$ and $b+c$, which span a hyperbolic sphere pencil that contains $Z_{a d}$. Thus

$$
\mu_{a d}:\left\{\begin{array}{ccc}
(a, b, c, d) & \mapsto & \left(d^{\prime}, c^{\prime}, b^{\prime}, a^{\prime}\right) \\
(A, B, C, D) & \mapsto & \left(D^{\prime}, C^{\prime}, B^{\prime}, A^{\prime}\right)
\end{array}\right.
$$

showing that $\mu_{a d}=\mu \rho_{a d}$ is one of the remaining symmetries.

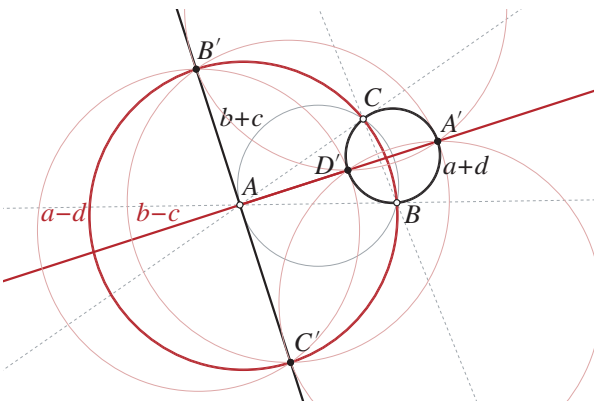

Fig. 13 As $a-d, b-c \perp a+d, b+c$

$\operatorname{span}\{a-d, b-c\}$ and $\operatorname{span}\{a+d, b+c\}$ define orthogonal elliptic and hyperbolic circle pencils.

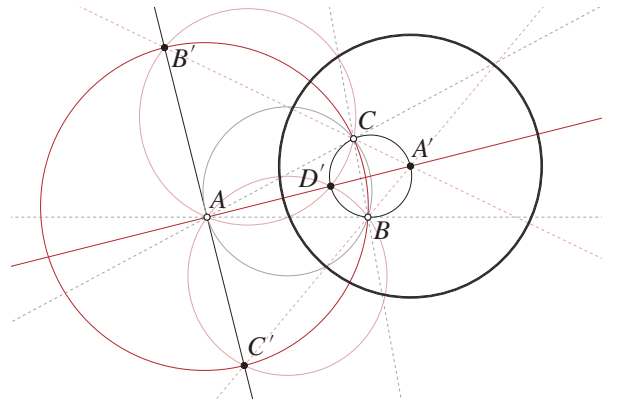

Fig. 14 The inversion $\mu_{a d}$ in the circle $Z_{a d}$ fixes the circles $a-d, b-c \perp Z_{a d}$ and exchanges the circles $a+d$ and $b+c$ of the hyperbolic pencil that $Z_{a d}$ belongs to; hence $\mu_{a d}:(A, B, C, D) \mapsto\left(D^{\prime}, C^{\prime}, B^{\prime}, A^{\prime}\right)$

Considering $\infty=-\frac{1}{R} D$ as the point at infinity, with the circum-radius $R<0$ as before, $Z_{a d}$ becomes a circle centred at $A^{\prime}=\mu_{a d}(D)$ and radius $r_{a d}$ given up to sign by

$$
r_{a d}^{2}=\frac{\left|Z_{a d}\right|^{2}}{\left\langle\infty, Z_{a d}\right\rangle^{2}}=-4 R r_{A},
$$

where $r_{A}>0$ is the ex-radius of the triangle as obtained from Lemma 3.3.

Note how similar this analysis is to that of the previous section. In fact, changing our mind about the orientations of the side $a$ of the triangle and of its circum-circle $d$, we would 
have arrived at $\left(D^{\prime}, C^{\prime}, B^{\prime}, A^{\prime}\right)$ as the dual of the original quadrangle, hence at the centre $Z_{a d}$ instead of $Z$ of the last section: four of the six angle bisectors would have changed to the complementary ones and the ex-circle $j_{A}$ would have taken the role of the in-circle; as a consequence, the inversion $\mu_{a d}$ would have taken the role of the antipodal map $\mu$.

By symmetry we thus obtain three inversions, $\mu_{a b}, \mu_{a c}$ and $\mu_{a d}$, in circles

$$
Z_{a b}=\frac{A+B-C-D}{2}, \quad Z_{a c}=\frac{A-B+C-D}{2}, \quad Z_{a d}=\frac{A-B-C+D}{2} .
$$

These circles intersect orthogonally, $Z_{a b} \perp Z_{a c}$ etc., so that the above inversions commute and satisfy

$$
\mu=\mu_{a b} \mu_{a c} \mu_{a d} .
$$

Hence we have obtained seven commuting Möbius involutions, $\mu, \mu_{x y}$ and $\rho_{x y}$, as symmetries of our configuration of dual quadrangles. These give rise to an eight element abelian symmetry group of the configuration:

Theorem 6.1. The 8-point configuration of a quadrangle consisting of

- a Euclidean triangle $(A, B, C)$ and the point at infinity, $D=\infty$, and

- the in- and ex-centres of the triangle, $D^{\prime}$ and $A^{\prime}, B^{\prime}, C^{\prime}$, respectively,

has an 8-element symmetry group whose non-trivial elements are the Möbius involutions

$\mu$ : the antipodal map, exchanging the two quadrangles,

$$
(A, B, C, D) \mapsto\left(A^{\prime}, B^{\prime}, C^{\prime}, D^{\prime}\right) ;
$$

$\mu_{x y}:$ inversion in a circle centred at an ex-centre and mapping the corresponding point to the in-centre;

$\rho_{x y}:$ hyperbolic rotation, by $\pi$, of the two ideal tetrahedra with ideal vertices

$$
(A, B, C, D) \text { and }\left(A^{\prime}, B^{\prime}, C^{\prime}, D^{\prime}\right),
$$

simultaneously interchanging the ideal vertices of each tetraheron in pairs.

This group exhausts the Möbius geometric symmetries of the configuration unless the cross ratio $q=[A ; B ; C ; D]$ satisfies one of the equations

$$
|q|^{2}=1, \quad|q-1|^{2}=1 \quad \text { or } \quad q+\bar{q}=1,
$$

in which case the triangle is isosceles, or $q$ satisfies all these equations, in which case the triangle is equilateral and the configuration has a full octahedral symmetry group of Möbius transformations.

Finally observe that, by considering the three tetrahedra with vertices $(A, B, C, D),\left(A^{\prime}\right.$, $\left.B^{\prime}, C^{\prime}, D^{\prime}\right)$ and $\left(Z_{a d}, Z_{a c}, Z_{a b}, Z\right)$ in projective 3 -space $\mathbb{R} P^{3}$, we obtain a desmic system, see $[3, \S 1]$ : the tetrahedra $(A, B, C, D)$ and $\left(A^{\prime}, B^{\prime}, C^{\prime}, D^{\prime}\right)$ are in fourfold perspective via $\mu_{x y}$ and $\mu$, with centres of perspective $Z_{x y}$ and $Z$. 


\section{References}

[1] Blaschke, W.: Vorlesungen über Differentialgeometrie III: Differentialgeometrie der Kreise und Kugeln. Springer Grundlehren XXIX, Berlin 1929.

[2] Hertrich-Jeromin, U.: Introduction to Möbius differential geometry. London Math. Soc. Lect. Note Ser. 300, Cambridge Univ. Press, Cambridge 2003.

[3] Hudson, R.W.H.T.: Kummer's quartic surface. Cambridge Univ. Press, Cambridge 1905.

[4] O’Hara, J.: Conformal dual of a quadruplet of points. Far East J. Math. Educ. 2 (2008), 1-11.

[5] Thurston, W.: The geometry and topology of three-manifolds. Lecture Notes 1980, Princeton Univ., electronic version 1.1 (2002).

Udo Hertrich-Jeromin

Institut für Diskrete Mathematik und Geometrie

Forschungsgruppe Differentialgeometrie und Geometrische Strukturen

Technische Universität Wien

Wiedner Hauptstraße 8-10/104

A-1040 Wien, Austria

e-mail: uhj@geometrie.tuwien.ac.at

Alastair King

Department of Mathematical Sciences

University of Bath

Bath, BA2 7AY, United Kingdom

e-mail: a.d.king@bath.ac.uk

Jun O’Hara

Department of Mathematics and Information Sciences

Tokyo Metropolitan University

Tokyo 192-0397, Japan

e-mail: ohara@tmu.ac.jp 